\title{
Changes in exercise habits and pulse wave velocity with lifestyle modification in Japanese
}

\author{
Nobuyuki Miyatake ${ }^{1}$, Noriko Sakano ${ }^{1}$, Takeshi Saito ${ }^{2}$, Takeyuki Numata ${ }^{2}$ \\ ${ }^{1}$ Department of Hygiene, Faculty of Medicine, Kagawa University, Kagawa, Japan \\ ${ }^{2}$ Okayama Southern Institute of Health, Okayama Health Foundation, Okayama, Japan \\ Email: miyarin@med.kagawa-u.ac.jp
}

Received 20 February 2012; revised 18 March 2012; accepted 2 April 2012

\begin{abstract}
We investigated the changes in exercise habits and brachial-ankle pulse wave velocity (baPWV) with lifestyle modification in Japanese. We used data for 105 men (48.2 \pm 13.8 years) and 110 women $(48.6 \pm$ 12.1 years) without any medications with a 1-year follow up. Subjects were given advice for dietary and lifestyle improvement. At the 1-year follow up, body weight and body mass index (BMI) were significantly decreased in both sexes. Abdominal circumference was significantly decreased in men. In addition, changes in exercise habits were noted in both sexes. In separate analysis in subjects without exercise habits at baseline, the changes in baPWV with exercise habits at follow up was lower than that without exercise habits at follow up in both sexes, but not at a significant level. Lifestyle modification may increase exercise habits, however, it did not evidently change baPWV in this population.
\end{abstract}

Keywords: Lifestyle Modification; baPWV; Exercise Habits

\section{INTRODUCTION}

Arterial stiffness represents one of the major hemodynamic factors determining pulse pressure even at an early stage of disease and its changes have been shown to be an independent predictor of hard endpoints in patients with a high cardiovascular risk. Pulse pressure and heart rate constitute other outcomes that may be useful as additional factors in risk assessment [1]. Pulse wave velocity (PWV) is measured from the initial upstroke of pressure wave and constitutes an established index of arterial stiffness. It is directly related to arterial compliance and other factors describing arterial stiffness [2]. PWV is not only a good tool for assessing vascular damage, but also an independent predictor of all-cause and cardiovascular mortality [3].
It is well known that regular physical activity has been shown to increase HDL and reduce resting blood pressure, triglycerides, abdominal fat accumulation, fasting blood sugar, and insulin responses to oral glucose challenge test [4-8]. The prevalence of subjects with exercise habits in Japan was reported to be $29.1 \%$ in men and $25.6 \%$ in women by the National Nutrition Survey in Japan [9]. Therefore, evaluation of the relationship between exercise habits and PWV may provide quite useful data for preventing future diseases in the Japanese general population.

In this study, we evaluated the changes in exercise habits and brachial-ankle PWV (baPWV) with lifestyle modification in Japanese without medications.

\section{SUBJECTS AND METHODS}

\subsection{Subjects}

We used data for 105 Japanese men $(48.2 \pm 13.8$ years $)$ and 110 Japanese women $(48.6 \pm 12.1$ years $)$ among 4200 subjects ( 1580 men and 2620 women), who met the following criteria: 1) received a health check-up including special health guidance (from April 2006 to August 2010) and a follow-up check-up 1-year later, 2) received anthropometric and exercise habits measurements as part of the annual health check-up, 3) received no medications for diabetes, hypertension, and/or dyslipidemia, and 4) provided written informed consent (Table 1).

At the first health check-up, all subjects were given instructions by well-trained medical staff on how to change their lifestyle as special health guidance. Nutritional instruction was provided with a well-trained nutriationist, who planned a diet for each subject based on their data and provided simple instructions (i.e. not to eat too much and to consider balance when they eat). Exercise instruction was also provided by a well-trained physical therapist, who encouraged each subject to increase their daily amount of steps walked.

Ethical approval for the study was obtained from the 
Table 1. Changes in parameters of enrolled subjects.

\begin{tabular}{|c|c|c|c|}
\hline & Baseline (Mean \pm SD) & Follow-up $($ Mean \pm SD $)$ & $p$ \\
\hline \multicolumn{4}{|l|}{ Men } \\
\hline Number of subjects & 105 & & \\
\hline Age & $48.2 \pm 13.8$ & & \\
\hline Height $(\mathrm{cm})$ & $170.5 \pm 6.0$ & & \\
\hline Body weight (kg) & $71.3 \pm 11.6$ & $70.0 \pm 11.1$ & 0.0003 \\
\hline Body mass index $\left(\mathrm{kg} / \mathrm{m}^{2}\right)$ & $24.5 \pm 3.7$ & $24.1 \pm 3.4$ & 0.0002 \\
\hline Abdominal circumference $(\mathrm{cm})$ & $85.6 \pm 10.2$ & $84.2 \pm 9.6$ & 0.0033 \\
\hline Hip circumference $(\mathrm{cm})$ & $95.1 \pm 6.6$ & $95.0 \pm 8.1$ & 0.8221 \\
\hline baPWV (right) $(\mathrm{cm} / \mathrm{s})$ & $1329.1 \pm 210.0$ & $1326.8 \pm 198.5$ & 0.8261 \\
\hline baPWV (left) (cm/s) & $1333.4 \pm 217.9$ & $1326.8 \pm 196.4$ & 0.5129 \\
\hline baPWV (mean) $(\mathrm{cm} / \mathrm{s})$ & $1331.2 \pm 212.4$ & $1326.8 \pm 195.2$ & 0.6529 \\
\hline \multicolumn{4}{|l|}{ Women } \\
\hline Number of subjects & 110 & & \\
\hline Age & $48.6 \pm 12.1$ & & \\
\hline Height (cm) & $157.5 \pm 5.0$ & & \\
\hline Body weight (kg) & $54.3 \pm 7.6$ & $53.7 \pm 7.5$ & 0.0171 \\
\hline Body mass index $\left(\mathrm{kg} / \mathrm{m}^{2}\right)$ & $21.9 \pm 3.1$ & $21.7 \pm 3.0$ & 0.0175 \\
\hline Abdominal circumference $(\mathrm{cm})$ & $76.4 \pm 8.7$ & $76.8 \pm 8.8$ & 0.5053 \\
\hline Hip circumference (cm) & $91.2 \pm 5.2$ & $90.6 \pm 5.1$ & 0.0623 \\
\hline baPWV (right) $(\mathrm{cm} / \mathrm{s})$ & $1241.1 \pm 189.1$ & $1231.9 \pm 182.8$ & 0.3532 \\
\hline baPWV (left) $(\mathrm{cm} / \mathrm{s})$ & $1258.6 \pm 198.7$ & $1254.3 \pm 206.0$ & 0.7391 \\
\hline baPWV (mean) $(\mathrm{cm} / \mathrm{s})$ & $1249.9 \pm 192.0$ & $1243.1 \pm 186.3$ & 0.5147 \\
\hline
\end{tabular}

Ethical Committee of Okayama Health Foundation.

\subsection{Anthropometric Measurements}

The anthropometric parameters were evaluated by using the following respective parameters such as height, body weight, body mass index (BMI), abdominal circumference, hip circumference. BMI was calculated by weight/ [height $]^{2}\left(\mathrm{~kg} / \mathrm{m}^{2}\right)$. The abdominal circumference was measured at the umbilical level and the hip was measured at the widest circumference over the trochanter in standing subjects after normal expiration [10].

\subsection{PWV Measurements}

The baPWV was measured using a form PWV/ABI (Colin, Co., Ltd., Komaki, Japan) after resting at least 15 minutes as described previously [11]. This instrument records PWV, blood pressure, electrocardiogram and heart sounds simultaneously. The subjects were examined in the spine position after at least 5 minutes rest, with electrocardiogram electrodes placed on both wrists, a microphone for detecting heart sounds placed on the left edge of the sternum, and cuffs wrapped on both the brachia and ankles. The cuffs were connected to a ple- thymographic sensor that determines volume pulse form and an oscillometric pressure sensor that measures blood pressure. Volume waveforms for the brachium and ankle were stored, and the sampling time was $10 \mathrm{~s}$ with automatic gain analysis and quality adjustment.

\subsection{Exercise Habits}

The data on exercise habits were obtained at interviews conducted by well-trained staff using the structured method of the National Nutrition Survey in Japan. The subjects were asked if they currently exercise (over 30 min per session, 2 times per week for duration of 3 months). When the answer was "yes", they were classified as subjects with exercise habits. When the answer was "no", they were classified as subjects without exercise habits.

\subsection{Statistical Analysis}

Data are expressed as means \pm standard deviation (SD) values. A comparison of parameters between the 2 groups was made using the unpaired $t$-test, $\chi^{2}$ test and covariance analysis; $p<0.05$ was considered to indicate statistical significance. 


\section{RESULTS}

The clinical parameters at the baseline and the 1-year follow up are summarized in Table 1. Body weight, BMI and abdominal circumference were significantly reduced with lifestyle modification after one year in men. Body weight and BMI were also significantly reduced after one year in women. However, baPWV was reduced after one year in both sexes, but not at a significant level.

We also evaluated the changes in exercise habits in enrolled subjects (Table 2). Subjects with exercise habits were significantly increased in both sexes.

The changes in parameters in subjects without exercise habits at baseline were also investigated (Table 3). With lifestyle modification, body weight, BMI and abdominal circumference were significantly reduced with lifestyle modification after one year in men. Body weight and BMI were also significantly reduced after one year in women. However, baPWV was also reduced after one year in both sexes, but not at a significant level.

Finally, among subjects without exercise habits at baseline, we further compared the change in baPWV between subjects with and without exercise habits at follow up. The changes in baPWV in subjects with exercise habits at follow up were lower than those in subjects without exercise habits at follow up in both sexes, but not at a significant level even after adjusting for age (men: $p=0.3204$, women: $p=0.3462$ ) (Table 4).

\section{DISCUSSION}

The main objective of this study was to explore the changes in exercise habits and baPWV in apparently healthy Japanese with lifestyle modification with a 1year follow up.

In some literatures, the link between exercise and PWV has been reported. Gando et al. have reported that

Table 2. Changes in exercise habits of enrolled subjects.

\begin{tabular}{|c|c|c|c|c|}
\hline & & \multicolumn{2}{|c|}{ Follow-up } & \multirow[b]{2}{*}{$p$} \\
\hline & & $\begin{array}{c}\text { Exercise } \\
\text { habits }(+)\end{array}$ & $\begin{array}{c}\text { Exercise } \\
\text { habits }(-)\end{array}$ & \\
\hline \multicolumn{5}{|l|}{ Men } \\
\hline \multirow{2}{*}{ Baseline } & Exercise habits $(+)$ & 36 & 6 & \multirow{2}{*}{0.0052} \\
\hline & Exercise habits (-) & 38 & 25 & \\
\hline \multicolumn{5}{|l|}{ Women } \\
\hline \multirow{2}{*}{ Baseline } & Exercise habits $(+)$ & 29 & 4 & \multirow{2}{*}{$<0.0001$} \\
\hline & Exercise habits (-) & 25 & 52 & \\
\hline
\end{tabular}

Table 3. Changes in parameters of enrolled subjects without exercise habits at baseline.

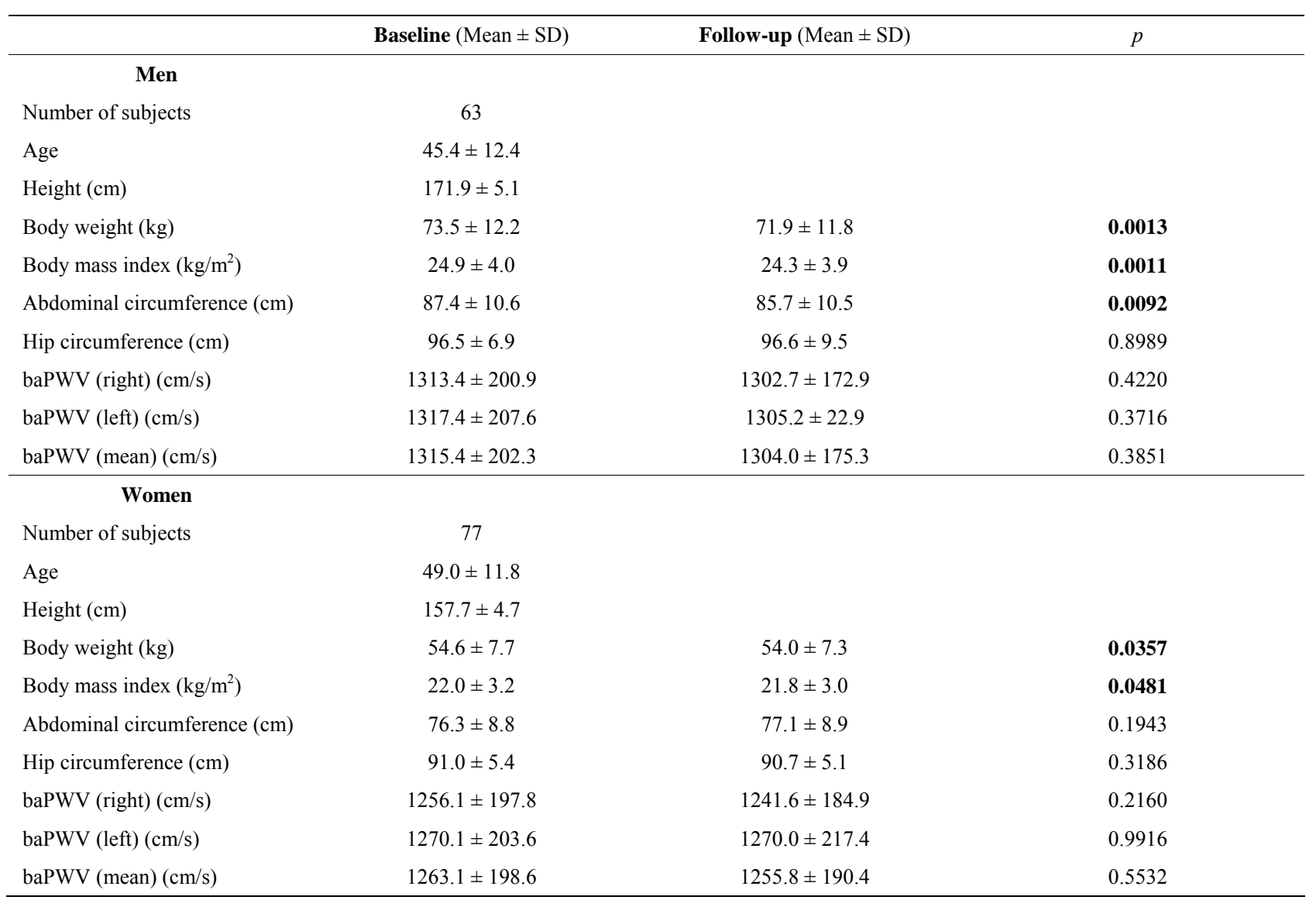


Table 4. Comparison of changes in baPWV between subjects with and without exercise habits at follow up (subjects without exercise habits at baseline).

\begin{tabular}{ccccc}
\hline & Number of subjects & Mean \pm SD & $p$ & $p$ (after adjusting for age) \\
\hline Men & 38 & $-21.0 \pm 99.0$ & 0.3749 & 0.3204 \\
Exercise habits $(-) \rightarrow(+)$ & 25 & $3.0 \pm 111.5$ & & \\
Exercise habits $(-) \rightarrow(-)$ & & & & 0.3462 \\
Women & 25 & $-29.1 \pm 101.1$ & 0.2206 & \\
Exercise habits $(-) \rightarrow(+)$ & 52 & $3.2 \pm 110.0$ & & \\
Exercise habits $(-) \rightarrow(-)$ & 52 &
\end{tabular}

longer time spent in light physical activity is associated with attenuation of arterial stiffening, especially in older people by cross-sectional analysis of 538 healthy Japanese [12]. Yamamoto K et al. also reported that baPWV was higher in poor-flexibility than in high-flexibility group [13]. In longitudinal analysis, Kawasaki et al. showed that a 6-month, twice-a-week exercise program emphasizing swimming significantly decreased baPWV in 11 men and 24 women [14]. Figueroa et al. also reported that a 12-week moderate-intensity combined circuit resistance and endurance exercise training improves arterial stiffness in 12 subjects [15]. In addition, homebased resistance training decreased baPWV in 12 healthy premenopausal women [16]. In this study, with lifestyle modification at an annual health check-up, subjects with exercise habits were significantly increased. Among subjects without exercise habits, the changes in baPWV in subjects with exercise habits at follow up was more decreased than that in subjects without exercise habits at follow up, but not at a significant level. Therefore, it seems reasonable to suggest that simply promoting exercise habits might result in decrease baPWV in some Japanese.

Potential limitations remain in our study. First, the small sample size in our study makes it difficult to infer causality between exercise habits and baPWV. baPWV did not significantly change with a 1-year follow up. Possibly, these results may come from low statistical power and/or misclassification of life-style modification. Second, we also could not reveal the mechanism of the linkage between exercise habits and baPWV. Insulin resistance is associated with arterial stiffness independent of obesity [17]. Exercise habits may improve insulin resistance and baPWV. The third, the 105 men and 110 women among 4200 subjects, all of whom wanted to change their lifestyle, underwent measurements for this study: they were therefore more health-conscious than the average person. Further prospective and large sample size studies are required in Japanese subjects.

\section{ACKNOWLEDGEMENTS}

This research was supported in part by Health and Labor Sciences
Research Grants from the Ministry of Health, Labor and Welfare, Japan.

\section{REFERENCES}

[1] Asmar, R., Darne, B., Assaad, M. and Topouchian, J. (2001) Assessment of outcomes other than systolic and diastolic blood pressure: Pulse pressure, arterial stiffness and heart rate. Blood Pressure Monitoring, 6, 329-333. doi:10.1097/00126097-200112000-00012

[2] Martyn, C.N. and Greenwald, S.E. (1996) Pulse wave velocity as a marker of vascular disease. Lancet, 348, 1586. doi:10.1016/S0140-6736(05)66200-9

[3] Laurent, S., Boutouyrie, P., Asmar, R., Gautier, I., Laloux, B., Guize, L., Ducimetiere, P. and Benetos A. (2001) Aortic stiffness is an independent predictor of all-cause and cardiovascular mortality in hypertensive patients. Hypertension, 37, 1236-1241.

[4] Miyatake, N., Takahashi, K., Wada, J., Nishikawa, H., Morishita, A., Suzuki, H., Kunitomi, M., Makino, H., Kira, S. and Fujii, M. (2003) Daily exercise lowers blood pressure and reduces visceral fat in overweight Japanese men. Diabetes Research and Clinical Practice, 62, 149157. doi:10.1016/S0168-8227(03)00176-1

[5] Miyatake, N., Nishikawa, H., Morishita, A., Kunitomi, M., Wada, J., Suzuki, H., Takahashi, K., Makino, H., Kira, S. and Fujii, M. (2002) Daily walking reduces visceral adipose tissue areas and improves insulin resistance in Japanese obese subjects. Diabetes Research and Clinical Practice, 58, 101-107. doi:10.1016/S0168-8227(02)00129-8

[6] Barnard, R.J., Ugianskis, E.J., Martin, D.A., Inkeles, S.B. (1992) Role of diet and exercise in the management of hyperinsulinemia and associated atherosclerotic risk factors. American Journal of Cardiology, 69, 440-444. doi:10.1016/0002-9149(92)90981-4

[7] Yamanouchi, K., Shinozaki, T., Chikada, K., Nishikawa, T., Ito, K., Shimizu, S., Ozawa, N., Suzuki, Y., Maeo, H., Kato, K., Oshida, Y. and Sato, Y. (1995) Daily walking combined with diet therapy is a useful means for obese NIDDM patients not only to reduce body weight but also to improve insulin sensitivity. Diabetes Care, 18, 775778. doi:10.2337/diacare.18.6.775

[8] Oshida, Y., Yamanouchi, K., Hayamizu, S. and Sato, Y. (1989) Long-term mild jogging increases insulin action despite no influence on body mass index or VO2 max. 
Journal of Applied Physiology, 66, 2206-2210.

[9] National Nutrition Survey in Japan (in Japanese) (2011). http:/www.mhlw.go.jp/houdou/2008/12/d1/h1225-5i.pdf

[10] Japanese Society of Internal Medicine (2005) Definition and the diagnostic standard for metabolic syndromeCommittee to evaluate diagnostic standards for metabolic syndrome. Nippon Naika Gakkai Zasshi, 94, 794-809. doi:10.2169/naika.94.794

[11] Kohara, K., Tabara, Y., Tachibana, R., Nakura, J. and Miki, T. (2004) Microalbuminuria and arterial stiffness in a general population: The shimanami health promoting program (J-SHIPP) study. Hypertension Research, 27, 471-477. doi:10.1291/hypres.27.471

[12] Gando, Y., Yamamoto, K., Murakami, H., Ohmori, Y., Kawakami, R., Sanada, K., Higuchi, M., Tabata, I. and Miyachi, M. (2010) Longer time spent in light physical activity is associated with reduced arterial stiffness in older adults. Hypertension, 56, 540-546. doi:10.1161/HYPERTENSIONAHA.110.156331

[13] Yamamoto, K., Kawano, H., Gando, Y., Iemitsu, M., Murakami, H., Sanada, K., Tanimoto, M., Ohmori, Y., Higuchi, M., Tabata, I. and Miyachi M. (2009) Poor trunk flexibility is associated with arterial stiffening. American Journal of Physiology: Heat and Circulatory Physiology,
297, 1314-1318.

[14] Kawasaki, T., Sullivan, C.V., Ozoe, N., Higaki, H. and Kawasaki, J. (2011) A long-term comprehensive exercise program that incorporates a variety of physical activities improved the blood pressure, lipid and glucose metabolism, arterial stiffness, and balance of middle-aged and elderly Japanese. Hypertension Research, 34, 1059-1066. doi:10.1038/hr.2011.81

[15] Figueroa, A., Park, S.Y., Seo, D.Y., Sanchez-Gonzalez, M.A. and Baek, Y.H. (2011) Combined resistance and endurance exercise training improves arterial stiffness, blood pressure, and muscle strength in postmenopausal women. Menopause, 18, 980-984. doi:10.1097/gme.0b013e3182135442

[16] Okamoto, T., Masuhara, M. and Ikuta, K. (2009) Homebased resistance training improves arterial stiffness in healthy premenopausal women. European Journal of Applied Physiology, 107, 113-117. doi:10.1007/s00421-009-1102-x

[17] Lee, J.W., Lee, D.C., Im, J.A., Shim, J.Y., Kim, S.M. and Lee, H.R. (2007) Insulin resistance is associated with arterial stiffness independent of obesity in male adolescents. Hypertension Research, 30, 5-11. doi:10.1291/hypres.30.5 